\title{
Scanning facility to irradiate mechanical structures for the LHC upgrade programme
}

\author{
Paul DERVAN $^{a}$, Richard FRENCH $^{b}$, Paul HODGSON $^{b}$, Hector MARIN-REYES $^{b}$, \\ Kerry PARKER $^{* b}$, John WILSON ${ }^{c}$, Matthew BACA ${ }^{c} \dagger$ \\ ${ }^{a}$ University of Liverpool $(G B),{ }^{b}$ University of Sheffield $(G B),{ }^{c}$ University of Birmingham $(G B)$ \\ E-mail: paul.dervan@cern.ch, richard.french@cern.ch, \\ phodgsonecern.ch, hector.marin.reyesecern.ch, \\ kerry.ann.parkerdcern.ch, john.allan.wilson@cern.ch, \\ matt.baca@cern.ch.
}

\begin{abstract}
The existing luminosity of the LHC will be increased in stages to a factor of 10 above its current level (HL-LHC) by 2022. This planned increase in luminosity results in significantly higher levels of radiation inside the proposed ATLAS Upgrade detector. This means existing detector technologies together with new components and materials need to be re-examined to evaluate their performance and durability at these higher fluences. Of particular interest is the effect of radiation on the upgraded ATLAS tracker. To study these effects a new ATLAS irradiation scanning facility has been developed using the Medical Physics Cyclotron at the University of Birmingham. The intense cyclotron beams allow irradiated samples to receive in minutes fluences corresponding to years of operation at the HL-LHC. Since commissioning in early 2013, this facility has been used to irradiate silicon sensors, optical components and carbon fibre sandwiches for the ATLAS upgrade programme. Irradiations of silicon sensors and passive materials can be carried out in a temperature controlled cold box which moves continuously through the homogenous beamspot. This movement is provided by a pre-configured XY-axis cartesian robot system (scanning system). This paper reviews the design, development, commissioning, performance results and future plans of the irradiation facility, fully operational since 2013.
\end{abstract}

Technology and Instrumentation in Particle Physics 2014,

2-6 June, 2014

Amsterdam, the Netherlands

* Speaker.

${ }^{\dagger}$ We are very grateful to Prof. D. Parker, Director of the Medical Physics Cyclotron, and his team, especially Mr. M. Smith, for setting up the proton beam and for their help in installing our apparatus. We also thank our technical colleagues in our home universities for their invaluable support. 


\section{Introduction}

By 2022 the luminosity of the LHC will have been increased in stages to approximately a factor of 10 above its current level. This planned increase in luminosity results in significantly higher levels of radiation within ATLAS and places stringent requirements on existing detector technologies. To study these effects a new bespoke ATLAS high intensity proton irradiation scanning facility has been constructed using the cyclotron at the University of Birmingham, enabling the

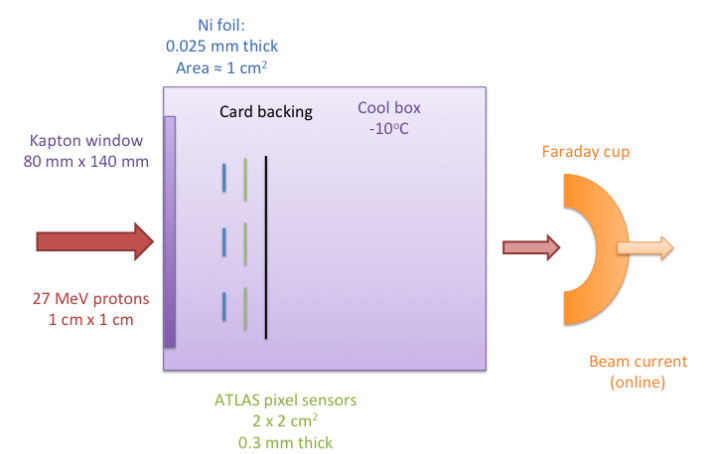

Figure 1: A schematic view of the beam layout used for irradiations evaluation of future detector technologies for the ATLAS upgrade. This can be done in several minutes as opposed to years of operation.

\section{The Birmingham Irradiation Facility}

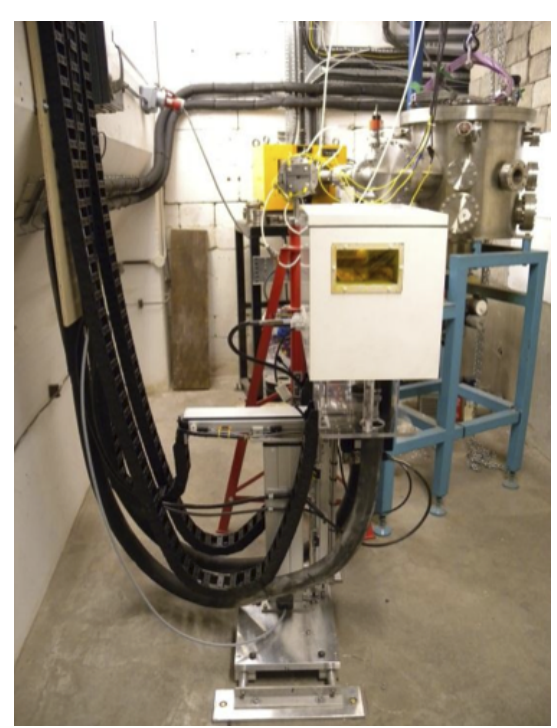

Figure 2: The pre-configured XY-axis Cartesian robot system and cool box within the high intensity irradiation area at the Medical Physics MC40 cyclotron, University of Birmingham
The irradiation facility has been commissioned at the University of Birmingham, using the Medical Physics MC40 cyclotron [1]. For the HL-LHC, fluences of approximately $1.0 \times 10^{15} 1 \mathrm{MeV} \mathrm{n}_{e q} \mathrm{~cm}^{-2}$ are required at the inner layer of the strip detector. Using $27 \mathrm{MeV}$ protons at the cyclotron, supplying an almost DC beam current, the protons have a Non-Ionising Energy loss (NIEL) enhancement factor of approximately 2. This means that a $1 \mu \mathrm{A}$ proton beam can achieve HL-LHC fluences of $10^{15} \mathrm{~cm}^{-2}$ in $80 \mathrm{~s}$ and with the possibility to achieve higher beam currents, makes the cyclotron beam an ideal facility for irradiation. The energy loss of a 27 $\mathrm{MeV}$ proton in $300 \mu \mathrm{m}$ of silicon is $1.1 \mathrm{MeV}$ and therefore the power dissipated in the sensor by a $1 \mu \mathrm{A}$ beam is $1.1 \mathrm{~W}$, suggesting that stacking sensors during irradiation should be avoided. The beam is collimated to 1 $\mathrm{cm}^{2}$, using pairs of horizontal and vertical plates, and additional cooling has been added to the collimators to allow currents of up to $2 \mu \mathrm{A}$.

The beam layout is shown schematically in figure 1. A pre-configured XY-axis Cartesian robot system (scanning table) and radiation hard cool box are used to scan the samples through the 27 $\mathrm{MeV}$ proton beam, see figure 2, where the beam is incident on an area within the $80 \times 140 \mathrm{~mm}^{2}$ box window, see [1] for more details. Samples are mounted on to a carbon fibre frame, and secured in place with cardboard and kapton tape. The frame is then attached to a matrix of fixing holes on the underside of the box lid. 
The temperature and humidity within the box are monitored by a PT100 sensor and controlled using a glycol heat exchanger system. When irradiating silicon sensors, it is crucial to maintain a low temperature within the box as radiation damage is temperature dependent and the sensors can overheat at high temperatures. Two fans are used to circulate the air within the box and the humidity is reduced with a dry $\mathrm{N}_{2}$ gas feed.

To check the beam profile and monitor the fluence received by the samples, three methods of dosimetry are employed, as described in [1], where irradiations at cyclotron energies are also shown to be equivalent to other facilities which use different energies and particle beams.

\section{Sample Testing}

Over 200 samples have been irradiated at the Birmingham irradiation facility. Variable beam currents of up to $2 \mu \mathrm{A}$ were used in order to achieve the required fluence. In figure 3, an IV measurement of pixel sensors at different fluences has been performed. The highest fluence, $5.0 \times 10^{15} \mathrm{n}_{e q} \mathrm{~cm}^{-2}$, used a beam current of $2 \mu \mathrm{A}$, and shows the effect of annealing; with a higher current than $1.0 \times 10^{15} \mathrm{n}_{e q} \mathrm{~cm}^{-2}$ sensor which was irradiated using a beam current of $1 \mu \mathrm{A}$. The silicon strip sensors can be characterised fur-

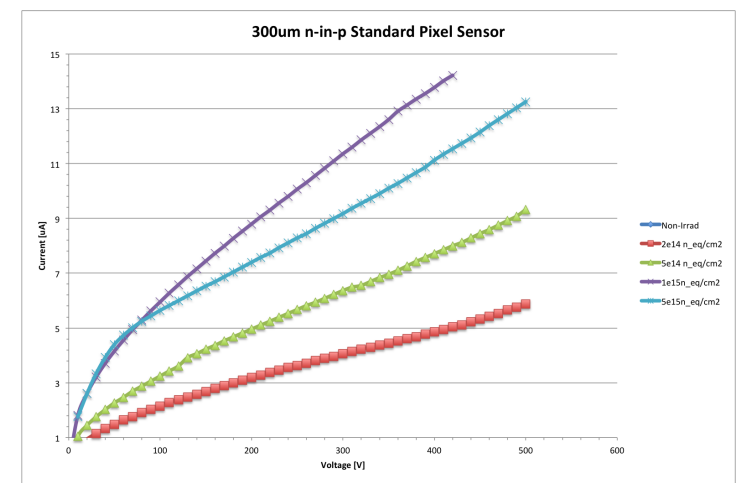

Figure 3: IV measurements of sensors irradiated at the Birmingham irradiation facility ther by charge collection efficiency (CCE) measurements which are performed using an Alibava [4] readout system with a ${ }^{90} \mathrm{Sr}$ source [2], whilst other samples are tested using other methods. The carbon fibre samples can undergo mechanical strength tests [5] and the electronic components can be visually analysed. Note however that the heating effects observed will not be significant at the HL-LHC where the fluence will be accumulated over years. However, the heating is significant when irradiating sensors at the Birmingham irradiation facility, and therefore further cooling is required.

\section{Prototype Cooling System}

The current cool box uses a glycol heat exchanger system with indirect air circulation, as described in section 2 and cools to $-10^{\circ} \mathrm{C}$ in approximately 2 hours. To prevent annealing, silicon samples are ideally kept below $0{ }^{\circ} \mathrm{C}$ during irradiations. However, at a beam currents above $1 \mu \mathrm{A}$, and based on the annealing in figure 3 , from further tests we can conclude that the sensors reached temperatures exceeding $100{ }^{\circ} \mathrm{C}$. The PT100 sensors within the cool box during irradiation are not in direct contact with the sensors and therefore no direct evidence of high temperatures has been recorded. Measurements suggest that the glycol chiller system is unable to remove enough heat from the sensors to prevent damage. A prototype for an improved cooling system with increased air circulation has been proposed and tested and a schematic of the system can be seen in figure 4 . The prototype system uses evaporative cooling by dripping liquid $\mathrm{N}_{2}$ (LN2) on to a block within 
the box. The block is placed in an overspill tray and thermally isolated from the bottom of the box to avoid damage from direct contact with the LN2. A fan is again used to circulate the cool gas around the box and a Norhof LN2 [3] dispensing unit, which controls the administration of small amounts of LN2, using the pressure within a 50 litre dewar to control the rate of flow. This unit is controlled via the Norhof GUI and maintains a set temperature within the cool box.

\section{Cooling Tests}

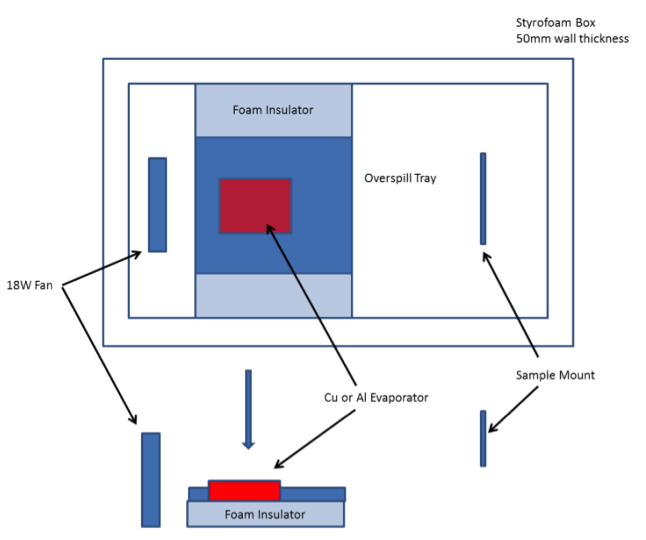

Figure 4: Prototype cool box schematic using LN2 dispensing system for evaporative cooling within the box
In order to test the system, a dummy cool box has been set up with the geometry in figure 4 , along with a $50 \Omega$ mini heater which uses a range of voltage to generate heat which simulates the different beam currents. This is applied directly on to a silicon sensor, attached to a PT1000 sensor to measure the temperature. Two additional PT1000 sensors are placed within the box, one from the LN2 system which is monitored in order to maintain the temperature within the box, and the other for an additional cross-check. The LN2 system has been set up to maintain the temperature of the cool box at $-48^{\circ} \mathrm{C}$. This is achieved in approximately 6 minutes. The materials used in the cool box have also been repeatedly immersed in LN2 to test their integrity and none show signs of damage.

The temperature of the sensor is recorded whilst applying $1.1 \mathrm{~W}$ to the sensor, using the mini heater, and whilst cooling, once the heater is switched off. The heating results can be seen in figure 5 and the maximum $\Delta \mathrm{T}$ which can be seen is $40^{\circ} \mathrm{C}$. However, the temperature never increases above $-5{ }^{\circ} \mathrm{C}$. Further tests are now needed, in particular measurements to optimise geometry. The box should also be purged with dry N2, as before, to avoid frosting.

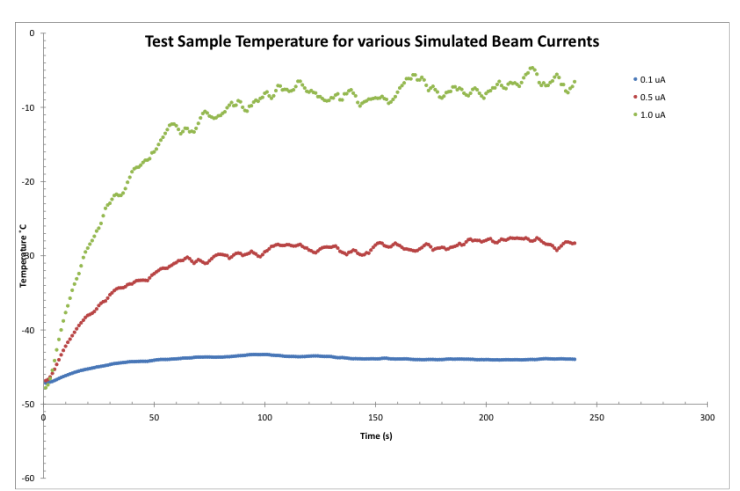

Figure 5: Temperature measurements of different simulated beam currents whilst applying heat to the sensor

\section{Conclusion}

The Birmingham irradiation facility has been fully commissioned and has irradiated a variety of samples, with further irradiations scheduled. The irradiations at cyclotron energies have been shown to be equivalent to other facilities, yet can achieve HL-LHC fluences on a much shorter time scale. Using variable beam currents allows samples to be irradiated to a range of fluences; however the higher beam currents show some evidence of overheating and it is therefore essential to improve cooling within the box during irradiation. A new prototype cooling system has been 
designed which uses a LN2 dispensing system and evaporative cooling. The system is easy to set up and use and reaches $-50{ }^{\circ} \mathrm{C}$ in 6 minutes compared to the current system which cools to a minimum of $-15^{\circ}$ in approximately 2 hours. Initial temperature tests show that applying heat equivalent to a $1 \mu \mathrm{A}$ beam with the new cooling system in a dummy cool box, the temperature plateaus at $-5^{\circ} \mathrm{C}$. The initial prototype is successful in removing heat generated by high beam currents, providing a suitable environment to irradiate silicon sensors.

\section{References}

[1] P. Dervan, R. French, P. Hodgson, H. Marin-Reyes, J. Wilson, The Birmingham Irradiation Facility, NIM A , 703 (2013), p.101

[2] P. Dervan, Silicon Strip Detectors for the ATLAS HL-LHC Upgrade, ATL-UPGRADE-PROC-2011-007 (2011)

[3] Norhof, LN2 microdosing system http://www.norhof.com/

[4] R. Marco-Hernanadez, IEEE Transactions on Nuclear Science NS-56 (2009), p.1642

[5] T. Jones, Measurements of the Flat-wise Tensile Strength of Irradiated and Non-irradiated Sandwich Test Tokens (2012) 